\title{
Une découverte inattendue
}

\section{Les archives audiovisuelles et cinématographiques du Sénégal}

\author{
Marco Lena et Tiziana Manfredi
}

\begin{abstract}
Citer cet article : Lena Marco, Manfredi Tiziana (2021), « Une découverte inattendue : les archives audiovisuelles et cinématographiques du Sénégal », Revue d'Histoire Contemporaine de l'Afrique, en ligne. URL : https://oap.unige.ch/journals/rhca/article/view/stclenamanfredi

Mise en ligne : 22 novembre 2021
\end{abstract}

DOI : https://doi.org/10.51185/journals/rhca.2021.e587

Le rêve de chaque historien est de trouver une archive où perdre et passer ses journées, de rester immergé dans des documents, de découvrir des sources historiques inédites et de pouvoir leur donner une nouvelle vie en les valorisant. Imaginons aussi qu'au moment de la trouvaille, I'historien en question n'est même pas en train de chercher des archives, mais le hasard lui permet de faire une découverte exceptionnelle qui dépasse le rêve et s'apparente, osons le dire, à un miracle. C'est dans une telle circonstance qu'en 2009, ma collègue vidéaste, Tiziana Manfredi et moi, comme historien et restaurateur audiovisuel, nous avons découvert l'existence des archives audiovisuelles du Service d'Informations (SI) du Sénégal, et depuis presque deux ans, nous avons pour objectif de donner, avec la Direction de la Cinématographie (DCl) sénégalaise, une nouvelle existence à ces images.

La "rencontre » avec ces archives s'est produite quand nous sommes entrés dans l'immeuble du ministère sénégalais de la Communication (situé à Dakar au Plateau, boulevard de la République) pour demander un permis de tournage. Ayant perdu l'orientation dans les locaux de ce grand bâtiment d'époque coloniale, nous nous sommes retrouvés devant la porte ouverte d'une pièce où étaient conservées les archives audiovisuelles du SI de l'ancien ministère de l'Information et de la Communication. L'image devant nos yeux était impressionnante : des milliers de bobines dont certaines au sol, et une lumière caravagesque qui éclairait la pièce, lui donnant une atmosphère d'immobilité ; la poussière renforçait la sensation d'être suspendu dans le temps. Cette magie qui se produisait devant nos regards fut brisée par la voix d'un technicien de la $\mathrm{DCl}$ qui nous exhortait à quitter l'endroit, car les visiteurs n'avaient pas l'autorisation de le traverser. Avant de quitter la pièce, nous avons demandé le contenu des bobines et la même voix nous répondit : " Ce sont les archives du service Cinéma... Malheureusement le passage au numérique a signé la fin des archives. Les techniciens qui pouvaient manipuler les bobines sont partis à la retraite et maintenant il n'y a personne pour les sauvegarder. Tout de même, nous cherchons des partenaires pour les sauver ensemble... ». 
Le temps a passé et j'ai décidé en 2015 de me former à Rome comme technicien restaurateur audiovisuel. Entre temps, Tiziana Manfredi et notre collaboratrice Ndeye Mane Touré, manager culturel, débutaient les échanges avec l'ancien directeur de la DCl, Hugues Diaz, pour réfléchir à une stratégie de mise en place d'un projet de sauvegarde et de valorisation de ces archives. H. Diaz nous expliqua qu'il voulait, depuis longtemps, sauver la mémoire audiovisuelle du Sénégal, mais que toutes les études réalisées par de potentiels partenaires de passage n'avaient jamais dépassé la phase de repérage. Je suis rentré à Dakar en 2017 pour me concentrer sur le projet en cours ${ }^{1}$ : il a finalement été écrit, après différentes versions, mais sans aboutir à une recherche de financements. Dans l'attente de trouver les fonds nécessaires, j'ai entamé une autre étude sur l'état des archives audiovisuelles de l'Institut Fondamental d'Afrique Noire (IFAN). Finalement, en 2019, nous avons décidé de ne plus attendre les financements pour le démarrage, car l'urgence était désormais trop insistante. C'est donc en novembre de la même année que nous avons récupéré 250 bobines des Actualités Sénégalaises des années 1960-1980, en accord avec H. Diaz et avec deux techniciens de la DCl.

\section{Les archives audiovisuelles et leurs enjeux}

\section{Découvertes des archives à Dakar et premières actions}

Dix ans après la première rencontre, nous sommes retournés sur les lieux. Depuis deux ans déjà, le bâtiment n'était plus en service et nous faisions face à une situation surréaliste. Le bâtiment, auparavant plein de vie, était devenu silencieux, complètement envoûté par d'épaisses couches de poussière et les locaux étaient presque complètement vidés de tous les objets et matériaux techniques, sauf ceux appartenant à l'époque précédente, soit les supports non numériques... Entre temps, les bobines avaient été déplacées au deuxième étage où Ndeye et Tiziana les avaient revues en 2017, lors d'un repérage avec $\mathrm{H}$. Diaz dans les locaux désaffectés. Deux ans plus tôt, avant de quitter le bâtiment, la $\mathrm{DCl}$ avait fait un tri entre ce qui semblait encore en bon état et ce qui sentait clairement le vinaigre ${ }^{2}$, produit par un phénomène de détérioration des matériaux organiques des supports analogiques - cette odeur saturait d'ailleurs les couloirs du quatrième étage où tout était entreposé, lors de la première rencontre. Ce dernier tri avait permis de placer le matériel récupérable dans des piles ordonnées dans une chambre vide.

Selon un inventaire effectué en 2012 par la DCl, seules 500 bobines sur les 6000 pouvaient être visionnées et sauvegardées. Sur ces 500 , seulement 51 avaient été cataloguées, avec des indications par rapport au format, au support et au contenu. Après dix jours de travail pour effectuer les tests d'acidité, 250 bobines n'étaient pas contaminées par le syndrome du vinaigre et une première liste nominative pouvait être dressée. Le reste, malheureusement, était abandonné sur place. Pendant ce temps, en explorant le reste du bâtiment, nous avons pu retrouver dans une première armoire des centaines de papiers et documents. Motivés par

\footnotetext{
${ }^{1}$ Après deux ans d'échanges à distance avec mes collègues Tiziana et Ndeye qui continuaient à dialoguer avec $\mathrm{H}$. Diaz, et deux expériences professionnelles dans les archives audiovisuelles - une à la Cinémathèque de Toulouse et la deuxième aux archives du Centre Cinématographique marocain (CCM) de Rabat -, où j'ai pu commencer à exercer ce métier sur les archives filmiques.

${ }^{2}$ Le syndrome du vinaigre est le phénomène de dépolymérisation spontanée qui se produit dans les pellicules photographiques et films cinématographiques, par dégradation de l'acétate en acide acétique, causant ainsi la détérioration du support des œuvres.
} 
cette découverte, nous avons poursuivi nos investigations et retrouvé les scripts des Actualités sénégalaises, des scenarii et autres documents à même de retracer la vie de l'ancien Bureau du Cinéma du SI. Nous avons finalement déplacé les bobines et ces documents dans les locaux actuels de la $\mathrm{DCl}$ qui a mis à disposition deux petites salles climatisées pour conserver les archives dans des conditions de stockage optimales.

La priorité était de se concentrer sur le fonds audiovisuel de l'ancien ministère de la Communication et de l'Information, qui rassemble notamment les Actualités sénégalaises produites depuis l'indépendance du pays jusqu'aux années 1980. Au mois d'avril 2020, nous sommes retournés dans l'ancien bâtiment ${ }^{3}$ et avons découvert une salle, au sixième étage, dédiée au service photo, où il y avait une vingtaine de placards pleins de négatifs de différents formats et époques, conservés dans des enveloppes et ayant subi apparemment moins d'altération. Cette deuxième collection, composée de plaques en verre, de négatifs $6 \times 6$ et $35 \mathrm{~mm}$, couvrait une période allant des années 1960 à 2001, ainsi qu'une documentation des années 1950: une vraie richesse pour comprendre la vie politique, sociale, économique et culturelle du Sénégal et compléter le fonds des archives du SI dans son ensemble.

Pour permettre le démarrage du projet, j'avais construit, avant de quitter l'Italie, une table visionneuse indispensable à la réparation et l'identification du contenu réel des bobines. Avec cet outil, deux étapes fondamentales pouvaient être entreprises. La première visait à établir la condition du support, la présence de perforation éclatées ou de coupures à même de mettre en danger la pellicule en la déchirant au moment de la numérisation ou du lavage. Ce contrôle minutieux est un préalable nécessaire à la seconde étape, consistant à vérifier le contenu de la bobine et à identifier précisément le sujet, car parfois le contenu de la pellicule ne correspondait pas au titre inscrit sur la boîte. Grâce à la Cinémathèque de Toulouse, quatre bobines ont été numérisées en $2 \mathrm{k}^{4}$. C'est de cette manière que les archives du SI ont pu reprendre vie et, qu'à travers un travail de valorisation de leur contenu, nous avons pu sensibiliser le grand public à la nécessité de sauvegarder la mémoire historique des archives audiovisuelles.

\section{Conserver les images: un enjeu pour l'histoire contemporaine de l'Afrique}

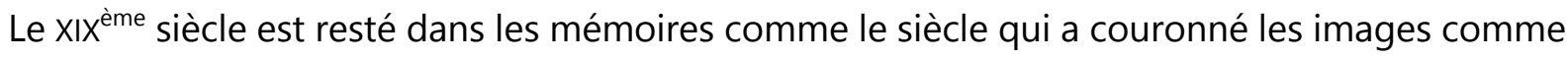
des sources incontournables. De simples attractions dans les expositions ${ }^{5}$, elles deviennent présentes au quotidien et un outil précieux dans la vie de chacun. À l'origine pourtant, presque personne, excepté le cinéaste Boleslaw Matuwieski, ne pouvait imaginer qu'un jour les images seraient une source historiographique importante et indispensable à la description de l'histoire mondiale. En effet, en 1897, ce cinéaste et ancien employé de l'usine des frères Lumière en France était censé filmer la visite du président français Félix Faure en Russie, où était aussi présent le chancelier allemand Otto von Bismarck. Cette visite aurait pu être considérée comme

\footnotetext{
${ }^{3}$ Le building de radiodiffusion fédérale, comprenant dix étages, construit dans les années 1950, était passé au ministère de I'Information après l'indépendance.

${ }^{4} 2 \mathrm{k}$ est un ordre de grandeur correspondant à une image de plus de 2000 pixel de large. Cette définition d'image est notamment utilisée par le cinéma numérique.

${ }^{5}$ Bancel Nicolas, Blanchard Pascal, Boetsch Gilles, Deroo Éric, Lemaire Sandrine (dir.) (2002), Zoos humains: de la Vénus hottentote aux reality shows, Paris, La Découverte.
} 
n'importe quelle autre, mais il y a des faits qui changent le monde, images à l'appui. Lors de cette visite, le chancelier allemand accuse en effet Félix Faure de ne pas avoir enlevé son chapeau en passant devant le drapeau russe, une faute qui aurait pu déclencher un incident diplomatique. Grâce au film de Matuwieski, le Tsar est à même de vérifier que le président français avait respecté la règle de conduite. Comme le souligne le cinéaste :

On a considéré que la projection d'un de ces clichés réfutait incontestablement une fausse déclaration venant de l'étranger, touchant à un comportement qui aurait été commis dans ces circonstances. Bien sûr, la chose avait peu d'importance, mais au final ce n'est qu'un exemple des services que la photographie animée peut rendre à la vérité, en vérifiant les témoignages d'hommes. C'est tout un côté anecdotique de l'histoire qui échappe désormais à l'imagination des narrateurs. ${ }^{6}$

Personne n'avait alors probablement réalisé le changement en cours et les images en mouvement devaient continuer de rester une attraction dans les expositions pour encore une décennie. Mais Matuwieski était convaincu qu'il était essentiel de collecter autant d'images du monde afin de pouvoir écrire une « histoire globale », telle qu'il l'appelait déjà. En 1898, il publie Une nouvelle source de l'histoire du cinéma pour soutenir sa thèse et écrire une histoire du monde :

Il suffira d'attribuer une section muséale, une étagère de bibliothèque, un cabinet d'archives aux répétitions cinématographiques qui auront un caractère historique. [...] Le prix de l'appareil de réception cinématographique comme celui des bandes pelliculaires, très élevé aux premiers jours, diminue rapidement et tend à tomber à la portée des simples amateurs de photographie. [...] Beaucoup d'entre eux, sans compter les professionnels, commencent à s'intéresser à l'application cinématographique de cet art et ne demandent pas mieux que de contribuer à constituer I'histoire. ${ }^{7}$

Avec le temps, l'idée de Matuwieski de conserver des archives cinématographiques historiques a été réalisée avec la création des cinémathèques et des archives audiovisuelles, qui permettent de découvrir et de connaître des faits historiques et des hommes politiques, quoique très inégalement répartis sur la planète. En se penchant sur des archives audiovisuelles africaines et en échangeant avec des historiens, on se rend facilement compte qu'il y a un manque de connaissances concernant les sources historiques provenant d'Afrique qui, en outre, sont peu valorisées. Les informations sur le continent ont longtemps été présentées à travers un regard extérieur et empreint du racisme de la vision coloniale. Ainsi, contribuer à sauver une partie des archives audiovisuelles du service cinéma du ministère de I'Information de la République du Sénégal est important : au-delà des images du Sénégal, cela conduit à conserver une partie méconnue de l'histoire mondiale.

\footnotetext{
${ }^{6}$ Matuszewki Boleslaw (1898), Une nouvelle source de l'histoire (Création d'un dépôt de cinématographie historique), Paris, p. 11. Pour plus de détails sur Matuszewki, voir Mazaraki Magdalena (2004), "Boleslaw Matuszewski : photographe et opérateur de cinéma », Revue 1895, 44, pp. 47-65.

${ }^{7}$ Matuszewki Boleslaw, Une nouvelle source..., op. cit., p. 10.
} 


\section{Le fonds des Actualités sénégalaises}

\section{Le Sénégal indépendant, pionnier de l'information audiovisuelle}

Le Sénégal a été l'un des premiers pays subsahariens à créer un service d'information utilisant le support cinématographique ${ }^{8}$. Léopold Sédar Senghor, premier Président, homme de culture mais aussi homme politique pragmatique, considérait le pouvoir des images comme utile à la consolidation de son pouvoir. Dès le premier jour après l'indépendance en 1960, le SI du bureau du cinéma est créé et dirigé par Paulin Soumonou Vieyra, I'un des premiers Africains subsahariens à avoir étudié à I'Institut des Hautes Études Cinématographiques (IDHEC) à Paris ${ }^{9}$ et à avoir réalisé, en 1955, un court métrage de 21 minutes intitulé Afrique sur Seine ${ }^{10}$, en collaboration avec Jaques Melo Kane, Mamadou Sarr et Georges Caristan. En dehors de Vieyra et Caristan, cameraman du court métrage précité, le Sénégal ne disposait pas d'un nombre suffisant de techniciens qualifiés dans le métier de l'audiovisuel, c'est pour cette raison que des techniciens français tels que Christian Lacoste et André Gaudier travaillèrent comme opérateurs et monteurs pour aider à la production d'actualités et de premiers films réalisés au Sénégal. Ainsi sont nées les Actualités sénégalaises, à partir de l'idée du ciné-journal français qui avait été, pendant des décennies, la seule source d'information diffusée dans les cinémas du Sénégal.

La structure est restée la même mais le contenu changea : pour la première fois les nouvelles traitaient d'indépendance et de progrès dans les domaines économique, technologique et politique, et surtout, les protagonistes étaient des Africains. Ainsi, pour la première fois, le spectateur se reconnaissait dans les images. Les nouvelles étaient hebdomadaires à l'exception des éditions spéciales, telles que la visite de chefs d'État étrangers ou les voyages à l'étranger de personnalités politiques importantes. La durée des séquences variait de 8 à 20 minutes et les nouvelles étaient projetées dans les différentes salles de cinéma du territoire national. L'importance de communiquer avec la population était si vitale que dans les régions où il n'y avait pas de salles de projection, un service de cinéma mobile avait été institué, grâce à l'utilisation de camions permettant de rejoindre les régions les plus reculées du Sénégal ${ }^{11}$. En utilisant des écrans montables et des projecteurs $16 \mathrm{~mm}$, les images du Sénégal indépendant pouvaient être vues par tous.

La structure des actualités peut être divisée en quatre parties, après un générique très court où un drapeau sénégalais flottait, avec le titre suivant en sous impression, "Le ministère de I'Information présente Les Actualités Sénégalaises». La première rubrique, Sénégal Aujourd'hui, avait pour objectif d'informer sur les principales nouvelles et avait comme protagoniste le Président ou un politicien. Sa durée était variable selon les informations, mais

\footnotetext{
${ }^{8}$ Voir le dossier thématique «Médias et décolonisations en Afrique (années 1940-1970), RHCA, 1, 2021, en ligne. URL: https://oap.unige.ch/journals/rhca/issue/view/01mediasdecolonisations (consulté le 16 novembre 2021).

${ }^{9}$ Pour plus de détails sur Vieyra, voir Pfaff Françoise (2004), «Paulin Soumanou Vieyra pionnier de la critique et de la théorie du cinéma africain », Présence africaine, 2(170), pp. 27-34.

${ }^{10}$ Ce film raconte la vie d'étudiants africains à Paris, leurs rencontres et la nostalgie qu'ils éprouvent loin de leur terre natale. L'Afrique est-elle aussi sur les bords de la Seine ? Ou au Quartier latin ? Interrogations aigres-douces d'une génération d'artistes et d'étudiants à la recherche de leur "civilisation", de leur culture, de leur avenir. C'est le premier court métrage tourné par des réalisateurs africains.

${ }^{11}$ Goerg Odile (2020), Un cinéma ambulant en Afrique: Jean-Paul Sivadier, entrepreneur dans les années 1950, Paris, L'Harmattan.
} 
chaque reportage durait presque deux minutes. La deuxième rubrique, Le Sénégal en marche, diffusait les nouvelles sur le développement économique, technologique et culturel. Elle n'était pas hebdomadaire mais mensuelle dans les Actualités sénégalaises. La troisième partie n'était pas produite au Sénégal mais en France, parce que le SI ne disposait pas de moyens suffisants pour une couverture mondiale. Ce service utilisait ainsi des informations tournées par les actualités françaises : elles abordaient la politique étrangère ou encore les événements sportifs en France et dans le monde - par exemple les Jeux Olympiques. Une quatrième partie (non systématique) consistait à informer les spectateurs sur divers événements du continent africain. La provenance de la production demeure obscure et pourrait être française, mais nous pouvons aussi envisager qu'il y eut un échange entre différents services d'informations africains, étant donné la présence d'une actualité camerounaise datant de 1962.

Le corpus des actualités est le premier fonds de la Cinémathèque nationale sénégalaise qui collectionna presque 6000 bobines dans lesquelles se retrouvent les Actualités sénégalaises, des films sénégalais et des documentaires éducatifs produits par I'UNESCO. Les Actualités sénégalaises représentent l'archéologie visuelle du Sénégal - un concept qui peut être étendu à toutes les actualités des pays africains nés après la colonisation - en tant que premières images indépendantes jamais produites dans ces territoires. Leur contenu, s'il est conservé et valorisé, fait entrer dans la postérité une période primordiale de l'histoire contemporaine de l'Afrique et du monde. La perte de ces sources historiques nous empêcherait de reconstituer les événements d'une époque riche en changements politiques, sociaux et culturels, alors que leur préservation et la recherche des négatifs originaux des bobines endommagées par le temps permettraient de fournir aux chercheurs, historiens et aux sociologues, des éléments précieux pour mieux comprendre l'évolution du Sénégal et de l'Afrique. La période qui suit l'indépendance reste en partie méconnue, et nous devons valoriser ces sources visuelles de la vie quotidienne pour mieux s'extraire d'un corpus d'images produites par des regards extérieurs.

Pour autant, l'idée de Matuwieski, qui considérait la fiabilité historique des images comme irréfutable, est à discuter, car celui-ci faisait référence à des images muettes, sans modification lors du montage. Comme tous les services de «propagande " nationale, les Actualités sénégalaises ne sont pas exemptes de manipulation de l'information ou déformation de la réalité, mais la sauvegarde de ces documents n'en reste pas moins indispensable - elles ont en outre permis aux Africains de se former à la production audiovisuelle. Les services des informations dans plusieurs pays ont en effet été utilisés comme « salle de musculation » pour apprenti-réalisateur et opérateur à la camera, comme ce fut le cas pour Baidy Sow et Momar Thiam au Sénégal, Souleymane Cissé au Mali et Edouard Sailly au Tchad. Cela est d'autant plus vrai au Sénégal, où le SI a contribué, grâce à la présence de Paulin Soumounou Vieyra comme Directeur de l'information, à la formation et au soutien de plusieurs réalisateurs ou producteurs cinématographiques. Ousmane Sembène à l'époque, connu comme écrivain, a pu réaliser son rêve de faire des films pour le continent africain, exclusivement grâce à la présence du réalisateur béninois à la direction du bureau du cinéma. II le rapporte lui-même dans une interview donnée à Présence Africaine en 2004 :

L'indépendance recouvrée, je rentre au Sénégal (de France) où je retrouve Paulin S. Vieyra au poste de chef du bureau du cinéma. Chaque semaine avec son équipe de cameramen, il préparait 
les actualités nationales Sénégal en marche. [...] L'idée me vient alors d'explorer notre continent dont je ne savais rien, en dehors de ma province. [...] De retour à Dakar, je dis à Paulin S. Vieyra mon intention d'aller apprendre à faire des films. Sa réponse fut directe : « C'est bien ; je suis là ». Ma formation terminée, je retourne au pays, [...] Paulin S. Vieyra m'aida à réaliser Borom Sarret, mon premier court métrage. [...] À l'occasion de ce cinquantenaire d'Afrique-sur-Seine, je me pose et me poserai toujours cette question : n'eussent été mes liens denses et profonds avec Paulin S. Vieyra, est-ce que j'aurais réalisé des films ?12

Ce propos est aussi relevé par Manthia Diawara13, car jusqu'aux années 1970, il n'y eut pas un seul film réalisé par des Sénégalais qui ne reçût l'aide des Actualités sénégalaises. Nous avons en effet retrouvé, dans les archives papier, divers documents qui témoignent de l'aide que le bureau du cinéma offrit aux productions cinématographiques sénégalaises. Par exemple, une partie du plan de tournage de Et la neige n'était plus de Ababakhar Samb Makharam, sur carte à en-tête des Actualités sénégalaises, ou encore la demande d'une caméra $35 \mathrm{~mm}$ pour le réalisateur mauritanien Med Hondo signée par Sembene Ousmane. Ce dernier document montre l'importance de l'action du bureau du cinéma, non seulement pour la réalisation nationale, mais aussi pour celle du continent.

\section{Dans la bobine "Le Sénégal au Festival mondial des arts nègres »}

Dans les 250 bobines sauvegardées et non affectées par le syndrome du vinaigre, il y a une bobine des Actualités sénégalaises sur la préparation du Premier Festival Mondial des Arts Nègres (FMAN) ${ }^{14}$ de 1966, tournée par Paulin Soumounou Vieyra. Cette bobine retrouvée représente un fonds historique fondamental pour imaginer la portée de ce festival pour le Sénégal - et pour la communauté « noire » dans le monde. Le Président Senghor, un des pères de la négritude, avait décidé que la « culture nègre » devait avoir un moment de glorification et être connue dans le monde entier. Pour cela, il prit la décision d'organiser un festival sur le continent africain et les cultures noires de la diaspora. Les Noirs pouvaient s'exprimer sans préjudice et se libérer du siècle de l'esclavage, de la colonisation et de la ghettoïsation culturelle. Une centaine d'artistes, écrivains, réalisateurs et intellectuels noirs provenant du monde entier rejoignirent Dakar en avril 1966 pour la transformer en capitale de la « culture négro-africaine ${ }^{15}$ ».

De cette bobine, tournée à l'origine en noir et blanc $35 \mathrm{~mm}$, il ne reste qu'une copie en $16 \mathrm{~mm}$, conservée dans les archives de la $\mathrm{DCl}$ à Dakar et d'une durée de 28 min. L'intention était de réaliser une carte postale de Dakar et du Sénégal, mais surtout d'expliquer à la population l'importance de cet événement, une première dans l'histoire du continent africain, et de faire de la capitale sénégalaise la ville de référence pour l'affirmation du Sénégal et de l'Afrique dans le monde entier. Les améliorations pour faire de Dakar une ville moderne, le dynamisme du port et les derniers travaux pour compléter l'aéroport (qui sera inauguré une semaine après la projection), les structures hôtelières, prêtes à accueillir visiteurs et artistes

\footnotetext{
12 Sembène Ousmane (2004), « Moment d'une vie : Paulin Soumanou Vieyra », Présence Africaine, 2(170), p. 22.

13 Manthia Diawara, né en 1953 à Bamako, est un écrivain et professeur de littérature d'origine malienne vivant aux États-Unis.

14 URL : https://fman.hypotheses.org/ (consulté le 17 novembre 2021).

15 Pour en savoir plus, lire Ficquet Éloi (2008), «L'impact durable d'une action artistique: le Festival Mondial des Arts Nègres de Dakar en 1966 », Africultures, 2(73), pp. 18-25.
} 
provenant du monde entier, sont mis en valeur. Dakar est décrite comme une ville à l'avantgarde. Conçues comme un voyage dans la production culturelle sénégalaise, les images illustrent la richesse de la danse, la capacité acquise de la production cinématographique, l'innovation du nouveau Musée dynamique, et le potentiel artistique de l'École des Beaux-Arts, sans oublier la librairie Présence Africaine, vraie plaque tournante pour la rencontre littéraire dans la capitale. Le Sénégal est aussi décrit comme un pays ou les différentes religions vivent en paix, dans un cadre d'une beauté naturelle prête à être découverte par le monde entier. Cinématographiquement parlant, cette actualité est un mélange entre des extraits montés des Actualités sénégalaises déjà réalisées et des images tournées pour l'occasion par le réalisateur de Porto Novo. Par exemple, une séquence présente le cinéma sénégalais avec Ousmane Sembène en train de réaliser le film Niaye ${ }^{16}$ : avec le support de l'archive papier, nous découvrons que le film a été tourné le 17 décembre 1964 par Vieyra à Keur Massar, un quartier de la banlieue de Dakar.

\section{Les archives papier}

La découverte des documents sur les Actualités sénégalaises et de scenarii de films nous a permis de mieux comprendre le fonctionnement du bureau du cinéma et d'identifier les techniciens engagés dans la production cinématographique. Cette découverte complète les informations pour chaque actualité dans le cas où les images ont été perdues. Mais étant donné qu'une partie des archives papier a été également perdue, c'est seulement à la fin du travail d'étude qu'il sera possible de rédiger un rapport complet sur ce fonds. Mises à part les données techniques sur les lieux, la durée, le réalisateur et la pellicule utilisée pendant le tournage, ces archives papier restituent la transcription de la voix qui accompagnait le reportage. Ceci permet d'en savoir plus sur la langue employée, d'analyser la manière dont l'information a été utilisée au Sénégal à cette époque et de mieux connaître le contenu des bobines.

Le document le plus ancien retrouvé date du 27 décembre 1960. C'est un script sur la donation d'un camion cinéma par le Centre Culturel Français au Service Cinéma de la République du Sénégal. En analysant le fonds photographique, nous avons retrouvé les images de ce camion sillonnant les routes, ce qui nous donne une indication, en image, de la façon dont s'effectuait le travail du service cinéma dans les régions. La langue utilisée dans les Actualités sénégalaises a été toujours le français, sauf pour le numéro 8/63 de février 1963 sur le Référendum ${ }^{17}$, avec une partie dans les différentes langues du Sénégal - du moins en wolof, pulaar, diola et toucouleur. Vu l'importance du sujet, le gouvernement voulait en effet s'assurer que le message soit compris par tout le monde. Nous avons retrouvé aussi des documents concernant le service de censure : dans un papier portant la signature de Paulin Soumounou Vieyra, il est notifié l'interdiction, sur le territoire sénégalais, de diffuser le film Terre Tropicale de Jean-Jacques Méhu datant de 1957, en raison d'une tonalité trop ouvertement coloniale.

\footnotetext{
${ }^{16}$ Court-métrage sénégalais de 1964 réalisé par O. Sembène, il s'agit d'une adaptation de la nouvelle Blanche-Genèse publiée par Présence africaine, qui raconte l'histoire d'un inceste commis par un chef de village à l'encontre de sa fille et son élimination par son fils, ancien tirailleur en poste en Indochine, au Maroc et en Algérie.

${ }^{17}$ Référendum constitutionnel sénégalais du 3 mars 1963 pour la nouvelle constitution qui supprime le poste de Premier ministre et établit un régime présidentiel.
} 
D'autres documents sur le contrôle cinématographique nous permettent de connaître la programmation dans les cinémas et ciné-clubs sénégalais.

\section{Les archives photographiques}

$\mathrm{Si}$, pour le matériel filmique, la perte de nombreuses bobines et scripts a rendu difficile le travail de reconstitution du fonds, les archives photographiques ont été retrouvées dans de meilleures conditions. L'archivage des photographies par année et avec métadonnées précisées sur les enveloppes - au sein de vingt placards - a facilité le travail d'étude. Grâce au registre où est consignée la totalité des reportages effectués et en croisant les données, nous pouvons affirmer que $95 \%$ de ce fonds photographique est consultable et en bon état. Seule l'année 1960 n'a pas été archivée dans un tiroir, mais dans des enveloppes éparpillées dans différents tiroirs : il n'y a pas une numérotation progressive par enveloppe mais par photo, manière de faire très tôt abandonnée à cause de la quantité importante de photographies réalisées, presque 5000 en deux ans. C'est en 1962 que le système d'archivage change : la numérotation par enveloppe permet d'identifier rapidement les années manquantes, mais empêche de connaître le nombre total de photographies dans les enveloppes et de repérer si certaines se sont perdues au fil du temps.

Le contenu de ce fonds, datant des années 1950 aux années 1980, est riche et les sujets sont hétérogènes et variés : visites de chefs d'États étrangers, de politiciens dans les régions, activités culturelles, événements religieux, sportifs, ou inauguration d'usines, de bateaux, vues de la ville de Dakar et d'autres villes, etc. Ici encore, ce fonds iconographique est indispensable pour écrire l'histoire moderne du Sénégal. Mais les identifications sur les enveloppes n'étant pas standardisées ${ }^{18}$, seule une étude complète du fonds permettra de révéler l'ensemble de son contenu. Une partie est datée des années 1950, mais en l'absence de registre et avec des métadonnées peu exhaustives, il est difficile de se rendre compte de l'ampleur des éléments illustrant la période sous administration française. Ces quelques 300 enveloppes renferment surtout des clichés de sujets tels que la société, l'architecture et des scènes de vie quotidienne en ville. Dans cette même section, nous dénombrons aussi une trentaine de plaques en verre, découverte très précieuse.

Il faut aussi mentionner l'existence d'une documentation sur les spectacles pendant le FMAN et sur le cinéma sénégalais; celle-ci a d'ailleurs été présentée dans le cadre d'une exposition qui s'est tenue en juin 2021 à Dakar dans les salles du Musée Théodore Monod. Dans ce fonds d'archives photographiques, on peut voir des images de nombreux chefs d'États africains de la première heure: Modibo Keita, Sékou Touré, l'Abbé Fulbert Youlou, Maurice Yaméogo, ainsi que d'autres dirigeants (par exemple la Reine d'Angleterre ou celle des PaysBas). Par ailleurs, le fait que Dakar ait été, depuis toujours, une destination prisée par les "stars » du spectacle, est confirmée par leur présence sur des négatifs : par exemple Ursula Andress en 1968, les Jackson Five en 1974, ou Mohamed Ali en 1981. Enfin, on trouve également des reportages sur les pèlerinages religieux dans trois sites importants de la vie spirituelle du Sénégal : Touba pour la confrérie mouride, Tivaouane pour la confrérie tidjane et Popenguine pour la communauté catholique. Ces images, très précieuses pour les chercheurs,

\footnotetext{
18 || est parfois indiqué le nom du sujet, d'autres fois sa fonction.
} 
pourront en outre servir, à travers des œuvres de valorisation à venir, à renforcer le sentiment de cohabitation pacifique en cette période actuelle, bien délicate pour le monde.

Redonner vie aux archives comporte aussi un travail de valorisation. Leur accès pour le grand public doit se penser en continu. La valorisation, à travers différentes actions et en utilisant divers langages artistiques, est un outil pédagogique qui peut faire changer le point de vue sur l'importance de l'archivage. Depuis novembre 201919, plusieurs moments de rencontres autour des archives ont été organisés, ainsi que la présentation d'une vidéoinstallation ${ }^{20}$ pendant le «Partcours », circuit d'art contemporain à Dakar21, a suscité un grand intérêt autour de la question de la préservation de la mémoire audiovisuelle et photographique grâce aux archives de la $\mathrm{DCl}$. Une présentation des extraits de quatre films numérisés pendant le festival de court métrage " Dakar Court » a permis d'échanger avec de jeunes réalisateurs et de souligner l'importance d'utiliser les images d'archives comme répertoire historique pour leurs réalisations. Cette rencontre, ainsi que l'exposition photographique sur le cinéma sénégalais de juin 2021, font partie des actions vitales pour ne pas faire retomber dans l'oubli la mémoire d'un passé récent au Sénégal, et contribuer à la connaissance de son histoire postindépendance.

Ces évènements ont suscité un grand intérêt : I'exposition photographique a été vue par environ 1000 visiteurs pendant un mois et l'enthousiasme était au rendez-vous. Toutes les activités ont été mises en place pour sensibiliser les institutions et le grand public sur l'importance de sauvegarder les archives et la mémoire audiovisuelle. Les prochaines étapes consisteront à la valorisation de ce patrimoine : avec la $\mathrm{DCl}$, nous sommes en train de créer un consortium des institutions qui conservent des archives pour pouvoir travailler ensemble et continuer l'œuvre de récupération et de mise à disposition de ces fonds.

\footnotetext{
${ }^{19}$ Matteo Maillard, "Au Sénégal, à la recherche des films perdus», Le Monde, 15 janvier 2020, en ligne. URL: https://www.lemonde.fr/afrique/article/2020/01/15/au-senegal-a-la-recherche-des-films-perdus_6026008_3212.html (consulté le 17 novembre 2021).

20 «Latent Frames » de Tiziana Manfredi et Marco Lena, 2020. L'installation vidéo propose un voyage dans l'espace de la mémoire ; elle revisite les lieux où ont été retrouvées les archives du patrimoine audiovisuel de la Direction de la Cinématographie en les faisant entrer en résonance avec des extraits vidéo et des photos provenant de ce même matériel analogique. Ressusciter des images du passé, un passé si concret dans la pensée contemporaine, nous permet de rouvrir un dialogue avec l'histoire. L'importance de ce patrimoine est soulignée, du fait que la mémoire nous interpelle, nous invite à l'écriture du présent et à une prospective du futur, où l'archive est une matière vivante et évocatrice dont on a besoin pour compléter les vides et les amnésies rétrogrades. En ligne. URL : https://flic.kr/s/aHsmSQKGvB (consulté le 17 novembre 2021).

${ }^{21}$ À Dakar, l'événement artistique du Partcours, initié en 2012, a lieu chaque année en décembre. L'organisation cherche à réunir le meilleur des espaces d'art professionnels de la capitale sénégalaise. La trentaine d'espaces membres est engagée toute au long de l'année dans la diffusion de l'art, de la culture et du savoir, à travers une grande variété de formes, et chacun reste libre de sa programmation pour le Partcours. Cette synergie des faiseurs d'art contemporain met en partage un itinéraire artistique à travers des espaces d'art majeurs, parfois insoupçonnés, dont les programmations débordent souvent dans les rues. Ils y présentent leur démarche de galeriste, de commissaire, d'artiste, offrant leur regard sur l'actualité de l'art et du débat artistique. En ligne. URL: http://www.partcours.art/ (consulté le 17 novembre 2021).
} 
Marco Lena

Association Atelier MamiWata, responsable scientifique du projet archives de la Direction de la Cinématographie du Sénégal Tiziana Manfredi Association Atelier MamiWata, directrice artistique du projet archives de la Direction de la Cinématographie du Sénégal

\section{Bibliographie}

Bancel Nicolas, Blanchard Pascal, Boetsch Gilles, Deroo Éric, Lemaire Sandrine (dir.) (2002), Zoos humains: de la Vénus hottentote aux reality shows, Paris, La Découverte.

FICQUET Éloi (2008), «L'impact durable d'une action artistique : le Festival Mondial des Arts Nègres de Dakar en 1966 », Africultures, 2(73), pp. 18-25.

GoERG Odile (2020), Un cinéma ambulant en Afrique: Jean-Paul Sivadier, entrepreneur dans les années 1950, Paris, L'Harmattan.

MAZARAKI Magdalena (2004), "Boleslaw Matuszewski : photographe et opérateur de cinéma », Revue 1895, 44, pp. 47-65.

Pfaff Françoise (2004), "Paulin Soumanou Vieyra pionnier de la critique et de la théorie du cinéma africain », Présence africaine, 2(170), pp. 27-34.

SEMBÈne Ousmane (2004), " Moment d'une vie : Paulin Soumanou Vieyra », Présence Africaine, 2(170), pp. 19-22. 